\title{
Influence of secondary decay on odd-even staggering of fragment cross sections
}

\author{
J. R. Winkelbauer, ${ }^{1}$ S. R. Souza, ${ }^{2,3}$ and M. B. Tsang ${ }^{1}$ \\ ${ }^{1}$ National Superconducting Cyclotron Laboratory and Department of Physics and Astronomy, Michigan State University, \\ East Lansing, Michigan 48824, USA \\ ${ }^{2}$ Instituto de Física, Universidade Federal do Rio de Janeiro Cidade Universitária, Caixa Postal 68528, 21941-972, Rio de Janeiro, Brazil \\ ${ }^{3}$ Instituto de Física, Universidade Federal do Rio Grande do Sul, Avenida Bento Gonçalves 9500, Caixa Postal 15051, \\ 91501-970, Porto Alegre, Brazil
}

(Received 6 March 2013; revised manuscript received 24 August 2013; published 23 October 2013)

\begin{abstract}
Odd-even staggering (OES) appears in many areas of nuclear physics and is generally associated with the pairing term in the nuclear binding energy. To explore this effect, we use the improved statistical multifragmentation model to populate an ensemble of hot primary fragments, which are then de-excited using the Weisskopf-Ewing statistical emission formalism. The yields are then compared to experimental data. Our results show that, before secondary decay, OES appears only in the yields of even mass fragments and not in the yields of odd mass fragments. De-excitation of the hot fragments must be taken into account to describe the data, suggesting that the OES in fragment yields is a useful criterion for validating or adjusting theoretical de-excitation models.
\end{abstract}

DOI: 10.1103/PhysRevC.88.044613

PACS number(s): 25.70.Pq, 24.60.-k

\section{INTRODUCTION}

Odd-even staggering (OES) is a widely observed phenomenon in nuclear physics. The early observed OES in nuclear masses associate the phenomenon with the pairing term in the binding energy $[1,2]$. Since particle production yields, including fission fragments, correlate strongly with the binding energy, measured fragment cross sections exhibit OES effects. Sawtooth-shaped charge correlations, based on the $Z$ distributions of fragments produced in different reaction mechanisms including fission and multifragmentation, have been reported [3-7]. Much of the previous work focused on staggering as a function of the atomic number, $Z$, because most experimental data were limited to only elemental identification.

Recently, isotopic identification for heavy reaction products can be achieved with state-of-the-art detectors. When the charge distributions are subdivided according to the neutron excess of the fragments, the staggering plots with isotope resolution reveal a more complex structure, suggesting that the de-excitation of the hot fragments contributes significantly to the observed OES effect. In fragments that are very neutron rich, the OES effect may be reversed; i.e., production of odd mass fragments are enhanced, compared to the less $n$-rich fragments. In this work, we investigate the OES effects of the isotopic fragment distributions obtained in the projectile fragmentation of ${ }^{40} \mathrm{Ca},{ }^{48} \mathrm{Ca},{ }^{58} \mathrm{Ni}$, and ${ }^{64} \mathrm{Ni}$ at $140 \mathrm{MeV} /$ nucleon, whose experimental analysis has been reported in Ref. [8].

Using the grand-canonical version of the improved statistical multifragmentation model (ISMM) [9] and the WeisskopfEwing statistical emission [10] to describe the de-excitation of the hot primary fragments, we examine the effects associated with the latter on the observed staggering. Our results suggest that the OES effect can be very useful in constraining the treatments adopted in the description of the de-excitation process. The remainder of the manuscript is organized as follows. In Sec. II we briefly recall the main features of the ISMM. The results are presented and discussed in Sec. III, and the conclusions are presented in Sec. IV.

\section{THEORETICAL FRAMEWORK}

In the framework of the grand-canonical approach [11,12], the yields $Y(A, Z)$ of a fragment with mass and atomic numbers, respectively, $A$ and $Z$, read

$$
Y(A, Z)=\frac{g_{A, Z} V_{f} A^{3 / 2}}{\lambda_{T}^{3}} e^{-\left[f_{A, Z}^{\text {smooth }}(T)-B_{A, Z}^{\text {paring }}-\mu_{p} Z-\mu_{b} A\right] / T},
$$

where $g$ stands for the spin degeneracy (taken as unit, except for the empirical values used for $A \leqslant 4), \lambda_{T}=\sqrt{2 \pi \hbar^{2} / m_{n} T}$ is the thermal wavelength, $m_{n}$ denotes the nucleon mass, $\mu_{p}$ $\left(\mu_{b}\right)$ represents the proton (baryon) chemical potential, and $f_{A, Z}^{\text {smooth }}(T)$ is the Helmholtz free energy associated with the fragment plus the pairing term of the binding energy $B_{A, Z}^{\text {pairing }}$. The free volume reads $V_{f}=\chi V_{0}$, where $V_{0}$ is the source's volume at normal density, and we use $\chi=2$ throughout this work. As in Refs. [11,12], the Helmholtz free energy has contributions from the fragment's binding energy $B_{A, Z}$, terms associated with the Wigner-Seitz correction [13] to the Coulomb energy $C_{c} \frac{Z^{2}}{A^{1 / 3}} \frac{1}{(1+\chi)^{1 / 3}}$, besides those related to the internal excitation of the fragment $f_{A, Z}^{*}$ :

$$
\begin{aligned}
f_{A, Z}^{\text {smooth }}(T)= & f_{A, Z}^{*}(T)-\left[B_{A, Z}-B_{A, Z}^{\text {pairing }}\right] \\
& -C_{c} \frac{Z^{2}}{A^{1 / 3}} \frac{1}{(1+\chi)^{1 / 3}} .
\end{aligned}
$$

where the pairing contribution $B_{A, Z}^{\text {pairing }}$ is subtracted from the binding energy $B_{A, Z}$, which makes $f_{A, Z}^{\text {smooth }}$ a smooth function of $(A, Z)$. For clarity, we use the standard SMM parametrization of the internal free energy [11], which is isospin independent, i.e., $f_{A, Z}^{*}(T)=f_{A}^{*}(T)$. The pairing term is written as

$$
B_{A, Z}^{\text {pairing }}=(-1)^{Z} \frac{\delta_{p}}{A^{\tau}} \frac{1}{2}\left[1+(-1)^{N-Z}\right],
$$

where $N=A-Z$ denotes the neutron number and $\delta_{p}$ is the pairing energy. In this work we employ the improved liquid 
drop model mass formula, labeled ILDM in Ref. [9], and we refer the reader to that work for the numerical values of the parameters. Although we have employed a liquid drop parametrization of the binding energy in all the calculations, we have checked that our conclusions are not affected by the use of more precise values of the binding energy, such as those given by the procedure described in Ref. [14].

With these definitions, $Y(A, Z)$ may be rewritten as the product of a smooth term multiplied by a rapidly oscillating function of $Z$ :

$$
Y(A, Z)=Y_{\text {smooth }}(A, Z) \times e^{(-1)^{Z} \frac{\delta_{p}}{A^{\tau}} \frac{1}{2 T}\left[1+(-1)^{N-Z}\right]},
$$

where

$$
Y_{\text {smooth }}(A, Z)=e^{-\left[f_{A, Z}^{\text {smooth }}(T)-\mu_{p} Z-\mu_{b} A\right] / T} .
$$

where one should notice that the exponential factor in Eq. (4) corresponds to $e^{B_{A, Z}^{\text {paring }} / T}$, which, together with Eq. (5), recovers Eq. (1).

The chemical potentials are determined by simultaneously solving the equations

$$
A_{0}=\sum_{A, Z} A Y(A, Z)
$$

and

$$
Z_{0}=\sum_{A, Z} Z Y(A, Z)
$$

where $A_{0}\left(Z_{0}\right)$ denotes the mass (atomic) number of the decaying source.

The de-excitation of the hot primary fragments is taken into account through the Weisskopf-Ewing statistical emission, as described in Refs. $[15,16]$. We consider the emission of nuclei up to ${ }^{20} \mathrm{O}$. For consistency, the same values of the binding energy $B_{A, Z}$ are used in the decay treatment. The same remarks hold for the density of excited states, as explained in Ref. [15]

\section{RESULTS}

The experimental $Z$ distributions obtained in the projectile fragmentation of ${ }^{40} \mathrm{Ca},{ }^{48} \mathrm{Ca},{ }^{58} \mathrm{Ni}$, and ${ }^{64} \mathrm{Ni}$ at $140 \mathrm{MeV} /$ nucleon on ${ }^{9} \mathrm{Be}$ reported in Ref. [8] are shown in Fig. 1, where the normalized quantity

$$
Y(Z)=\sum_{A} Y(A, Z) / \sum_{A, Z} Y(A, Z)
$$

is plotted for each case. The data, represented by the full circle, increases, on the average, as a function of the fragment's atomic number $Z$. A detailed inspection reveals that $Y(Z)$ deviates up and down from the local average value. As observed before, this staggering is weaker in the reactions with more neutron-rich projectiles.

The predictions made by the ISMM, presented in Sec. II, are also displayed in this figure and are represented by the full (primary yields) and dashed (final yields) lines. The model is primarily used to give insights into the OES, so there is no attempt to fit the OES magnitude in the data with the calculation. Instead of doing calculations with different values of temperature in order to account for the variation of

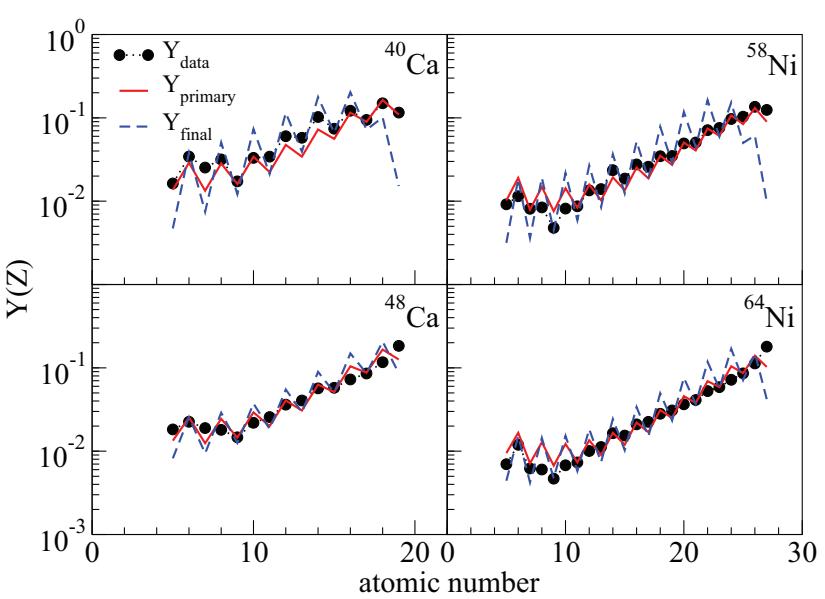

FIG. 1. (Color online) $Z$ distribution of fragments observed in the projectile fragmentation of several projectiles on a ${ }^{9} \mathrm{Be}$ target. The data are taken from Ref. [8] whereas the calculations correspond to the grand-canonical version of the ISMM, using the improved liquid drop formula of Ref. [9], at breakup temperature $T=4.0 \mathrm{MeV}$. For details, see the text.

the centrality of the collisions, for clarity and simplicity, we adopted a single average value of $T=4.0 \mathrm{MeV}$ for all systems. This is the standard strategy adopted in statistical calculations since the properties of the system before de-excitation cannot be unambiguously assessed, as many important aspects would have to be taken for granted. In the present case our procedure is further justified by the fact that Ref. [5] shows that the staggering magnitude is fairly insensitive to the excitation energy and/or the shape of the charge distribution. Therefore, using slightly different average temperatures would not affect the conclusions of this paper. Furthermore, although the prompt breakup assumed in the SMM might not be the best representation of the de-excitation mechanism at the selected temperature, the good qualitative agreement between the model predictions and the experimental results displayed in Fig. 1 suggests this choice is reasonable. This also corroborates the findings of Ref. [17], which shows that the GEMINI++ code [18] and the SMM predict fairly similar $Z$ distributions, except at extremely low excitation energies. Therefore, this aspect should not impact our conclusions. The model results follow the experimental trend of exhibiting weaker staggering effects in the case of the neutron-rich projectiles. Furthermore, by comparing the primary and final distributions, one sees that the deviations are clearly enhanced by secondary decays.

To isolate the local staggering behavior, we follow the procedure from Ref. [4]. We obtain the average value $\bar{Y}_{Z}(Z)$ by carrying out a parabolic fit, considering five points from $Z-2$ to $Z+2$. When $Z$ is close to or at either end of the range of the experimental data, the points lying outside the data are obviously not used. More specifically, five points are always employed in the fit; one simply shifts the selected region so that it does not extend beyond the edges. The ratio

$$
R_{Z}(Z)=Y_{Z}(Z) / \bar{Y}_{Z}(Z)
$$

is plotted in Fig. 2, for the data from Ref. [8] shown in Fig. 1. There is a systematic uncertainty $(\approx 10 \%)$ in the original 


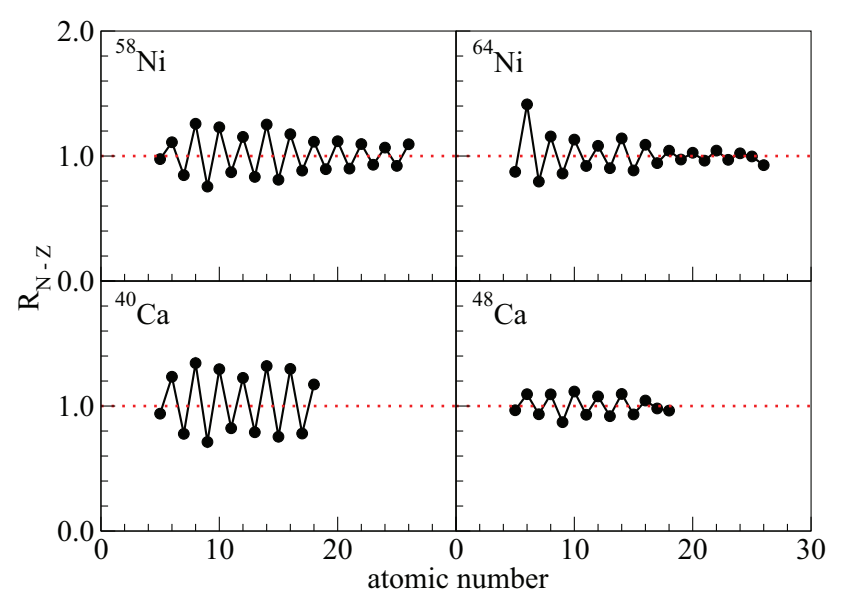

FIG. 2. (Color online) Ratio between $Y_{Z}(Z)$ and $\bar{Y}_{Z}(Z)$ for the four projectiles in Ref. [8].

data that comes mainly from the transmission efficiency of the fragment separator and the beam intensity normalization. These errors are approximately canceled out by using a ratio in the present analysis, so that the uncertainties of the plotted ratios are about $1-2 \%$ and are omitted for clarity. The features of this figure are consistent with previous studies. There is a weaker effect for the neutron-rich projectile, and the trend generally decreases with increasing $\mathrm{Z}$.

In the literature, the staggering effects have been associated with the pairing energy, $\delta_{p}$. According to Eq. (4), the staggering effects would be different depending on whether the neutron excess, $N-Z$, is even or odd. In the ISMM framework, the only nonsmooth contribution to the primary yields is the empirical binding energies. This is illustrated in Fig. 3 for the ${ }^{58} \mathrm{Ni}$ system. To explore this issue, we subdivide the experimental yields by their neutron excess and plot even and odd values separately. The quantity

$$
Y_{N-Z}(Z)=Y(A, Z) / \sum_{Z} Y(A, Z),
$$

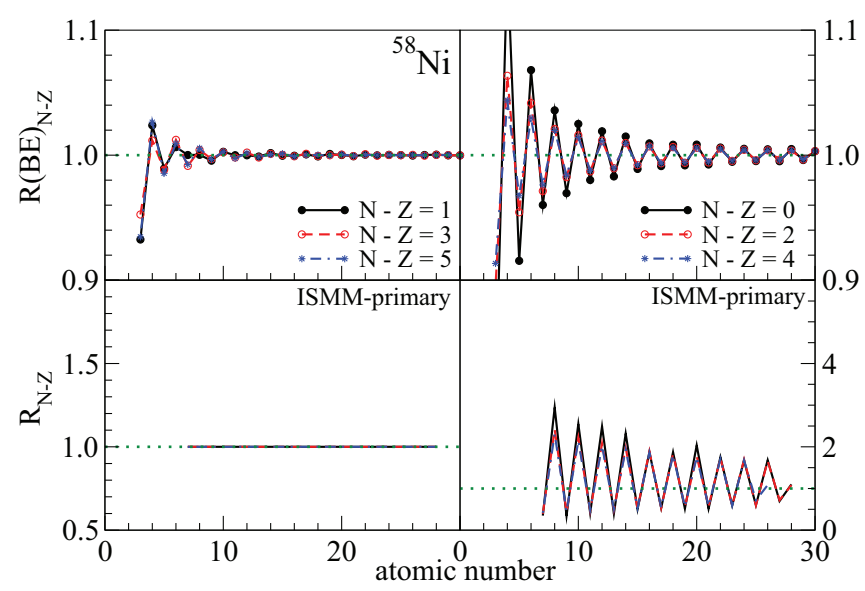

FIG. 3. (Color online) Comparison between staggering behavior in experimental binding energies (top panels) and primary yields from the ISMM (bottom panels). Fragments with odd neutron excess are shown on the left panels, while fragments with even neutron excess are shown on the right. gives the yields of an isotope $Z$ with neutron excess $N-Z$. Based on Eq. (4), the staggering effect should be absent for odd $N-Z$, whereas important staggering effects should be expected when $N-Z$ is even due to the multiplying factor $e^{(-1)^{Z} \frac{\delta p}{A^{\tau}} \frac{1}{T}}$

The ratio

$$
R_{N-Z}(Z)=Y_{N-Z}(Z) / \bar{Y}_{N-Z}(Z)
$$

is plotted in the bottom panels of Fig. 3 for odd and even $N-Z=1,3,5$ (left panels) and $N-Z=0,2,4$ (right panels), obtained from the primary yields of the ISMM. For succinctness, we focus on the ${ }^{58} \mathrm{Ni}$ projectile, but similar results are also obtained with the other projectiles. As expected from Eq. (4), the primary yields from ISMM clearly show that the OES is not observed in the case of odd neutron excess values, whereas the OES is observed for even $N-Z$ and the staggering diminishes as $Z$ increases. Aside from the different behavior for odd and even $N-Z$, the staggering effects seem to be independent of the $N-Z$ values. This behavior is consistent with the empirical binding energies, as illustrated in the top panels of Fig. 3, where we plot the same type of ratio as above, but using the binding energies

$$
R(B E)_{N-Z}(Z)=B E_{N-Z}(Z) / \overline{B E}_{N-Z}(Z) .
$$

Figure 3 shows that staggering in the yields of fragments with odd neutron excess is not related to the primary phase of multifragmentation.

Since most of the hot primary fragments have already decayed prior to being observed at the detectors, we use the implementation of the Weisskopf-Ewing statistical emission described in Refs. $[15,16]$ to estimate the effects of the deexcitation process on the fragment yields. The corresponding ratios are exhibited in the top panels of Fig. 4. One notes that the fragments' de-excitation enhances the deviations from the average values so that $R_{N-Z}$ now exhibits fluctuations on the same order of magnitude as those observed in the experimental

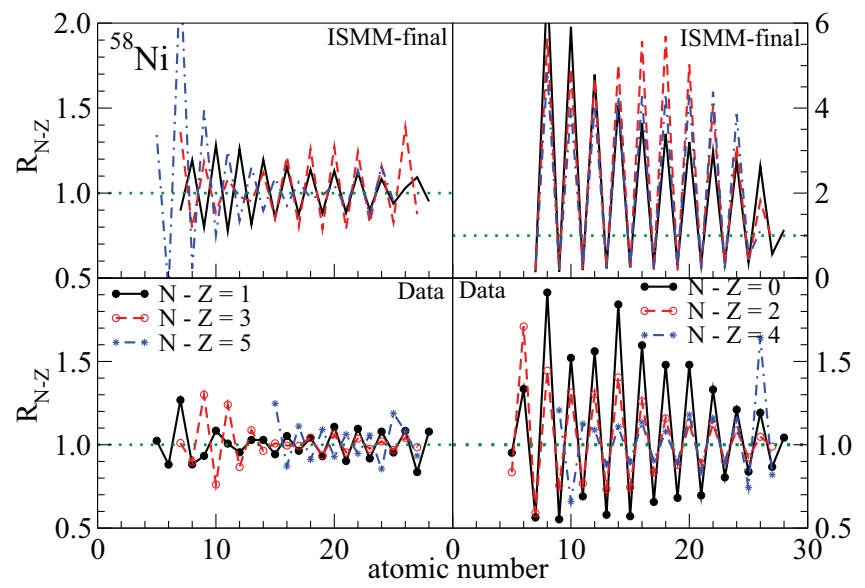

FIG. 4. (Color online) The results obtained using the ISMM with Weisskopf-Ewing de-excitation are displayed in the top panels, and the experimental results obtained with the projectile fragmentation data reported in Ref. [8] are shown in the bottom panels. Odd values of neutron excess are exhibited in the left panels, whereas even values are displayed in the right. For details, see the text. 


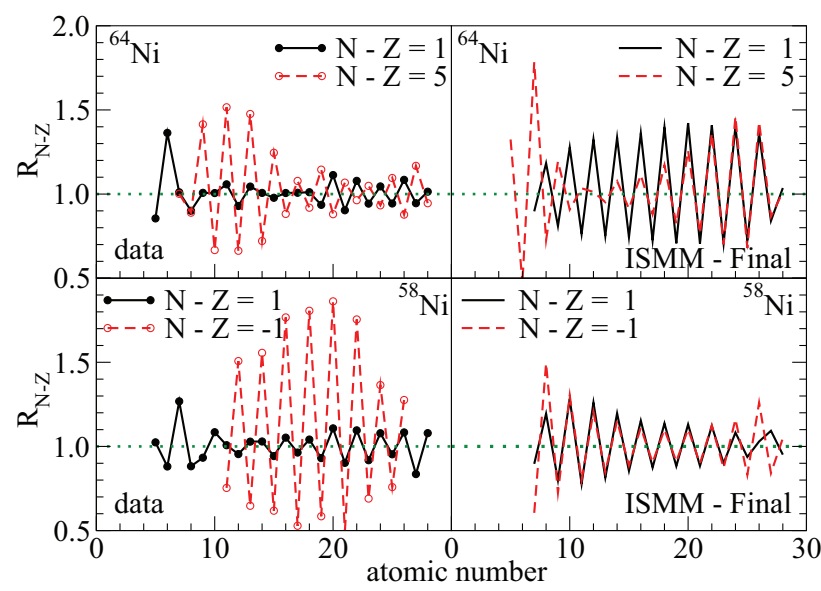

FIG. 5. (Color online) Comparison of the ratios obtained with $N-Z=-1$ and $N-Z=1$ for the ${ }^{58} \mathrm{Ni}$ projectile (lower panels) and with $N-Z=1$ and $N-Z=5$ in the case of the ${ }^{64} \mathrm{Ni}$ projectile (upper panels). The experimental ratios are calculated using the yields of Ref. [8]. For details, see the text.

data for odd $N-Z$. However, the fluctuations are much larger in the case of even values of neutron excess, about twice the experimental values. One should note that the scale used in the top right panel of Fig. 4, corresponding to the model results for even neutron excess, is different from that adopted in the other panels of the figures. Thus, although in the framework of the ISMM the de-excitation of the primary fragments is absolutely necessary to reproduce the experimentally observed staggering effects in the case of odd $N-Z$, it leads to fluctuations which are much larger than those actually observed in the data for fragments with even $N-Z$. Therefore, it strongly suggests that $R_{N-Z}$ is very sensitive to the de-excitation scheme used in the model calculations and this observable could be used to constrain the treatment for the decay of excited fragments.

There is a flip in the odd-even staggering for $N-Z=5$ isotopes in the ${ }^{58} \mathrm{Ni}$ projectile data plotted in the bottom left panel of Fig. 4; i.e. the cross sections of odd- $Z$ nuclei are enhanced relative to those with even- $Z$ nuclei. This effect is more clearly exhibited in the data obtained from the neutronrich projectile, ${ }^{64} \mathrm{Ni}$, as shown in the top panels of Fig. 5 ( $R_{5}$, joined by dashed lines). In addition, in contrast to the trend exhibited in even $N-Z$ isotopes where the staggering effects decrease with neutron richness, the magnitude of $R_{5}$ is much larger than $R_{1}$, for the lighter elements $(Z<14)$. The magnitude of the staggering effects in $R_{5}$ decreases and becomes similar to $R_{1}$ at higher $Z$. This is in agreement with the staggering observed in neutron-number distributions for neutron-rich projectiles [7]. This behavior cannot be easily explained by the ISMM since the primary yields lead to $R_{N-Z}=1$ for odd $N-Z$, as shown in the bottom left panel of Fig. 3. In the framework of this model, the OES can only be explained by intricate correlations associated with the deexcitation of the primary fragments, as one sees in the top right panel of Fig. 5, which shows $R_{N-Z}$ computed with the final yields. However, the reversed OES takes place only at small $Z$ values, while it is observed in the data over the whole $Z$ region.

Another striking feature observed in the experimental data is the amplification of the staggering in the distribution associated with neutron-deficient fragments $(N-Z=-1)$ compared with the ratios obtained with neutron-rich ones $(N-Z=1)$. The corresponding experimental observations are shown in the bottom left panel of Fig. 5, for the ${ }^{58} \mathrm{Ni}$ projectile. This amplification has no explanation in the framework of the ISMM since Eq. (4) predicts that the primary yields would be strictly smooth for $N-Z= \pm 1$. To check whether the de-excitation treatment employed in this work could account for the observed enhancement, the ratios obtained with the final ISMM yields are displayed in the bottom right panel of Fig. 5. This enhancement is not seen in the model calculations. Clearly, more detailed investigations are needed to understand this feature.

\section{CONCLUDING REMARKS}

We have examined the effects of the de-excitation on the staggering observed in the yields of the fragments produced in projectile fragmentation. The ISMM qualitatively explains this feature through odd-even effects associated with the pairing term of the nuclear binding energy. However, we have found that the ISMM is not able to reproduce the experimentally observed odd-even staggering effects present in the case of fragments with odd $N-Z$, if the effects associated with the de-excitation of the hot primary fragments are not taken into account. Even though the sequential decay fixes this shortcoming of the model, it leads to fluctuations which are much larger than observations in the case of fragments with even $N-Z$. Further theoretical investigation might lead to an improvement in the understanding of the de-excitation process. For example, this model currently assumes that the pairing term in the binding energy has no temperature dependence, a dependence which would affect how the hot fragments de-excite. Further examination of the staggering effect exposes additional deficiencies of the models employed in this work, as the enhancement of the ratio obtained with neutron-deficient $(N-Z=-1)$ fragments compared to that calculated with neutron-rich $(N-Z=1)$ nuclei cannot be reproduced theoretically. Moreover, the phase shift in the staggering behavior for $N-Z=1$ and $N-Z=5$ leads to a flip at large $Z$. This is only partially reproduced by the model, which predicts a flip at small $Z$. OES contrasts with observables used in the past in that it depends qualitatively on the de-excitation phase of the model, instead of only quantitatively [14,17]. Indeed, although no staggering is predicted by the grand-canonical ensemble in the primary stage for odd $N-Z$, it appears when the decay of the primary fragments is taken into account. Although the primary treatment will influence the final results, the study of OES seems to be particularly useful to constrain the treatments employed in the description of the decay of the primordial hot fragments as it affects the OES both quantitatively and qualitatively.

\section{ACKNOWLEDGMENTS}

We acknowledge CNPq, FAPERJ BBP grant, FAPERGS, and the joint PRONEX initiatives of CNPq/FAPERJ under Contract No. 26-111.443/2010 for partial financial support. This work is supported by the US National Science Foundation under Grant No. PHY-1102511. 
[1] W. A. Friedman and G. F. Bertsch, Phys. Rev. C 76, 057301 (2007).

[2] W. A. Friedman and G. F. Bertsch, Eur. Phys. J. A 41, 109 (2009).

[3] M. V. Ricciardi, A. V. Ignatyuk, A. Keliç, P. Napolitani, F. Rejmund, K.-H. Schmidt, and O. Yordanov, Nucl. Phys. A 749, 122 (2005).

[4] M. D’Agostino, M. Bruno, F. Gulminelli, L. Morelli, G. Baiocco, L. Bardelli, S. Barlini, F. Cannata, G. Casini, E. Geraci, F. Gramegna, V. L. Kravchuk, T. Marchi, A. Moroni, A. Ordine, and Ad. R. Raduta, Nucl. Phys. A 861, 47 (2011).

[5] G. Casini et al., Phys. Rev. C 86, 011602 (2012).

[6] E. Galichet, M. F. Rivet, F. Gagnon-Moisan, B. Borderie, M. Colonna, and R. Roy, EPJ Web Conf. 31, 00017 (2012).

[7] I. Lombardo et al., Phys. Rev. C 84, 024613 (2011).

[8] M. Mocko et al., Phys. Rev. C 74, 054612 (2006).

[9] S. R. Souza, P. Danielewicz, S. Das Gupta, R. Donangelo, W. A. Friedman, W. G. Lynch, W. P. Tan, and M. B. Tsang, Phys. Rev. C 67, 051602(R) (2003).
[10] V. F. Weisskopf and D. H. Ewing, Phys. Rev. 57, 472 (1940).

[11] S. R. Souza, M. B. Tsang, R. Donangelo, W. G. Lynch, and A. W. Steiner, Phys. Rev. C 78, 014605 (2008).

[12] S. R. Souza and M. B. Tsang, Phys. Rev. C 85, 024603 (2012).

[13] J. P. Bondorf, R. Donangelo, I. N. Mishustin, C. Pethick, H. Schulz, and K. Sneppen, Nucl. Phys. A 443, 321 (1985).

[14] W. P. Tan, S. R. Souza, R. J. Charity, R. Donangelo, W. G. Lynch, and M. B. Tsang, Phys. Rev. C 68, 034609 (2003).

[15] S. R. Souza, R. Donangelo, W. G. Lynch, and M. B. Tsang, Phys. Rev. C 76, 024614 (2007).

[16] S. R. Souza, M. B. Tsang, B. V. Carlson, R. Donangelo, W. G. Lynch, and A. W. Steiner, Phys. Rev. C 80, 041602 (2009).

[17] S. R. Souza, B. V. Carlson, R. Donangelo, W. G. Lynch, and M. B. Tsang, Phys. Rev. C 88, 014607 (2013).

[18] R. J. Charity, M. A. McMahan, G. J. Wozniak, R. J. McDonald, L. G. Moretto, D. G. Sarantites, L. G. Sobotka, G. Guarino, A. Pantaleo, L. Fiore, A. Gobbi, and K. D. Hildenbrand, Nucl. Phys. A 483, 371 (1988). 